\title{
Finite Element Modeling and Wave Propagation Analysis for Lens-Less Line Focus Acoustic Microscopy
}

\author{
Guorong Song, Dengqian Qin, Yan Lyu, Guangfu Hong, Yuyang Xu, Bin Wu and \\ Cunfu He \\ College of Mechanical Engineering and Applied Electronics Technology, Beijing University of Technology, Beijing, \\ China.
}

\begin{abstract}
(Received 29 January 2016; accepted 26 April 2016)
A finite element method for simulation of lens-less line focus acoustic microscopy is proposed in this paper to nondestructively evaluate the leaky surface wave (LSW) velocity. The defocusing measurement model is established, in which the geometrical focusing radius will be $20 \mathrm{~mm}$. The piezoelectric polyvinylidene fluoride film is selected as the active element. The excitation is a standard black Harris wavelet signal with a centre frequency of $5 \mathrm{MHz}$. Simulations of measurements on typical bulk materials (Al) are carried out. Then, the time-resolved wave signal series are acquired when the defocusing distance varies continuously. The LSW velocity will be easily determined by the examination of the slope of the LSW's arrival time versus the defocusing position. The LSWs' propagating path will be analyzed geometrically in time-domain. Meanwhile, the LSWs' velocities are also extracted by applying the specially developed digital signal processing algorithm to the defocusing experimental data, which is called $V(f, z)$ analysis method based on two-dimensional fast Fourier transform. Finally, the relationship between the time-resolved method and the $V(f, z)$ technique is discussed, in which the interpretation of the formation of surface waves and the description of its analysing methods will be given.
\end{abstract}

\section{INTRODUCTION}

Finite element method (FEM) ${ }^{1}$ has been developed rapidly and used extensively for scientific exploration and engineering analysis. Numerous researches have been done validating the approach and methodology. Many efforts have been leading to the possibility of excellent correlation between simulation and experiment. Hence, the FEM is in dominant position in the process of experiment design.

The mechanical performance plays a vital role in material sciences. However, few newly developed materials, such as nano-fabricated materials, metal glass, size-limited specimens, etc., can be examined by the conventional destructive methods., ${ }^{2,3}$ Thus, in recent years, many researchers contributed great efforts to the evaluation of mechanical properties, such as nano-indentation, acoustic microscope, laser ultrasonic, etc $^{4-7}$ Atalar $e t a l{ }^{8}$ firstly monitored the variation of the amplitude of the transducer output voltage $V$ as a function of spacing $\mathrm{Z}$ from specimen toward acoustic lens, namely the $V(z)$ curve. The $V(z)$ curve oscillates periodically and depends on the elastic properties of the reflecting surface uniquely. Later, Weglein and Wilson ${ }^{9}$ reported the periodicity of dips appearing in $V(z)$ curves. Parmon and Bertoni ${ }^{10}$ yielded a clear physical picture of signal formation in the reflection acoustic microscope and provided a simple formula to determine the LSW velocity. The next significant move toward quantitative measurements of anisotropic materials was taken by Kushibiki and colleagues, ${ }^{11}$ who invented the line-focus-beam (LFB) technique by means of measuring the interference phenomena in $V(z)$ curves.

Based on a time-domain Green's function formalism, D. Xiang and $\mathrm{N}$. N. Hsu ${ }^{12}$ designed a large aperture lens-less linefocus transducer for material characterization by simultaneously measuring the surface wave velocities and the bulk wave transit times, from which the thickness and anisotropy may be deduced. Guorong Song, et al. ${ }^{13,14}$ developed an ultrasonic measurement system for non-destructive determination of elastic constants by this time-resolved method (TRM). The system adopted a line focus PVDF transducer to implement the non-destructive experiments by measuring the elastic wave velocities, in which the directly reflected echoes and the reradiated Rayleigh waves are time resolved during transducer defocusing. ${ }^{15}$ Typical waves used for bulk material evaluation are longitudinal waves, transverse waves, and Rayleigh surface waves. In this case, the leaky surface wave and the specular reflection wave are separated in time domain and the velocities would be easily determined from the time delay. However, it should be mentioned that the first directly reflected echoes will be shifted to line up, so that the slope of the Rayleigh echoes can be used in calculation of the surface wave velocity.

In this paper, we will focus on the evaluation of the leaky surface wave (LSW) velocity of materials from the twodimensional finite element simulation of a line focus PVDF transducer using PZFlex (Weidlinger Associates Inc, USA) software package. First, the defocusing measurement model is established, in which the geometrical focusing radius will be $20 \mathrm{~mm}$. The directly reflected echo (DRE), the leaky surface wave (LSW) reradiated to the PVDF film, and the bottom reflected echo (BRE) are clearly time resolved. The LSW velocity would be determined by the examination of the slope of the LSW's arrival time versus the defocusing position. Then, the LSWs' propagating path will be analysed geometrically in time-domain. Meanwhile, the LSWs' velocities are also extracted by applying the specially developed digital signal processing algorithm to the defocusing experimental data, which is called $V(f, z)$ analysis method based on two-dimensional fast Fourier transform (2-DFFT). Finally, the relationship between the TRM and the $V(f, z)$ technique is discussed, in which the interpretation of the formation of surface waves and the understanding of its analysing methods will be given in de- 


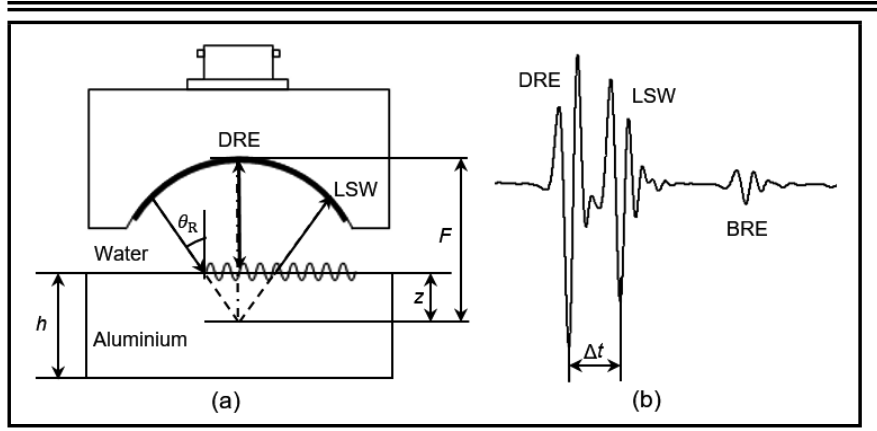

Figure 1. Schematic of time-resolved pulse/echo measurement.

tails.

\section{THEORETICAL ANALYSIS}

The time-resolved method was first developed by Hsu's group. ${ }^{12,16}$ Xiang $^{12}$ measured the LSW velocity in time domain using a lens-less line focus acoustic transducer with a broadband pulse excitation.

The wave propagating paths are shown in Fig. 1a. Here, $F, z, h$ are the focal length, the defocusing distance, and the sample's thickness, respectively. Next, $\Theta_{R}$ is the critical angle of the water/sample interface. At any arbitrary defocus position, the DRE, LSW, and BRE will appear in one waveform, as shown in Fig. 1b, which is a typical analogue signal. As it shows, the pulsed signals are clearly time resolved. $\delta t$ denotes the time delay of the LSW versus the DRE. A series of waveforms can be obtained while the defocusing distance $\mathrm{z}$ varies continuously.

The LSW velocity can be determined by measuring the linearity (slope $m=z / \delta t$ ) of the time interval between the LSW and DRE with the defocusing distance $z$. When $m$ is obtained, the LSW velocity can be evaluated through the following equation: ${ }^{12,17}$

$$
C_{R}=C_{\mathrm{W}}\left[1-\left(1-\frac{C_{\mathrm{W}}}{2 m}\right)^{2}\right]^{-\frac{1}{2}} ;
$$

where $C_{\mathrm{w}}$ is the longitudinal wave velocity in the coupling fluid, usually water. This method also holds for broadband pulse excitation. So, if one can determine every $\delta z$ at every single frequency included in the bandwidth, a series of consecutive surface wave velocities can be obtained by $V(f, z)$ technique. Lee, et al. ${ }^{18}$ explored the principles of this $V(f, z)$ measurement by a 2-D FFT waveform processing method, and applied it on the dispersion curves of thin plates. First, assuming that $v(t)$ is the time history of the transient wave function, the DRE signal can be represented as $v_{0}(t)$. For a nondispersive case, the LSW can be denoted as $v_{1}(t)$. Meanwhile, the BRE signal will not affect the interference phenomenon in $V(z)$ curve. Cleary, $v$ is a function of defocusing position $z$. Finally, we can get the waveform representation in terms of $t$ and $z$ :

$$
v(t, z)=v_{0}(t, z)+v_{1}(t, z) .
$$

It can be observed that $v_{1}(t)$ and $v_{0}(t)$, which are also function of defocusing distance $z$, only differ in phase and amplitude. Then we obtain:

$$
v_{1}(t, z)=g(z) \cdot v_{0}\left(t-m^{\prime} z, z\right) ;
$$

where $g(z)$ is an amplitude factor as a function of defocusing distance $z$, and $m^{\prime}$ is the ratio of time delay at different $z$

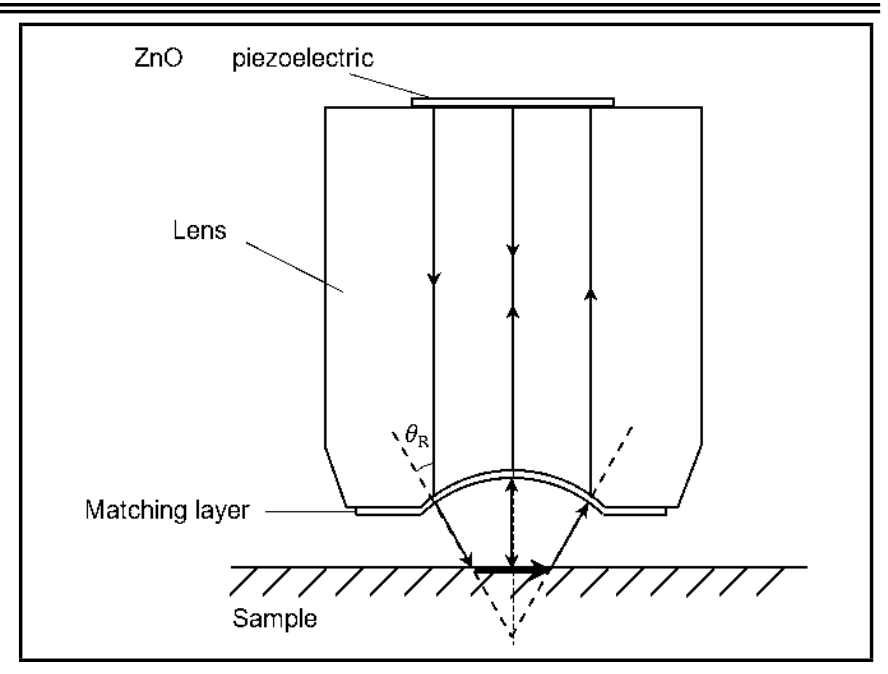

Figure 2. Schematic of conventional acoustic microscope with lens.

$\left(m^{\prime}=1 / m\right)$. Also, for a non-dispersive measurement, $m^{\prime}$ is a constant. So we get:

$$
v(t, z)=v_{0}(t, z)+g(z) \cdot v_{0}\left(t-m^{\prime} z, z\right) .
$$

Fourier transform Eq. (4) to frequency domain:

$$
V(f, z)=V_{0}(f, z)+g(z) \cdot V_{0}(f, z) e^{-i \cdot 2 \pi f \cdot m^{\prime} z} .
$$

For a certain frequency $f$, the amplitude of $|V(f, z)|$ is a periodic function of $z$, and the oscillating period ought to be written as:

$$
\Delta z=\frac{1}{f \cdot m^{\prime}} .
$$

Substitute $m^{\prime}=1 / m$ and Eq. (6) into Eq. (1), the wellknown equation in acoustic microscopy ${ }^{11}$ comes up

$$
C_{\mathrm{R}}=C_{\mathrm{W}}\left[1-\left(1-\frac{C_{\mathrm{W}}}{2 f \cdot \Delta z}\right)^{2}\right]^{-\frac{1}{2}}
$$

In dispersive case (thin plates, coatings, etc.), the slope $m$ will be also dispersive and non-linear, i.e. $m=m(f)$. So, Eq. (1) will not be used. In other words, the TRM can only be utilized in non-dispersive measurements under pulseecho mode, while, usually, conventional acoustic microscopy works in single frequency tone-burst mode. ${ }^{12}$ Figure 2 shows the schematic of conventional acoustic microscope with lens. This inspires us that, if the time-resolved measurements using broadband pulses are applied on dispersive materials and analysed by $V(f, z)$ method, a series of dispersion curves of guided waves will be obtained. However, there still exists one basic problem: why Eq. (7) used in conventional acoustic microscopy (with a focus lens) can be applied in time-resolved measurements (using PVDF without lens)? They differ a lot in geometry. Hence, a FEM model is established to illustrate the wave propagating path from every exciting angle segment of the PVDF. Furthermore, the $V(f, z)$ technique is derived from both dispersive and non-dispersive wave measurement, while TRM is derived from non-dispersive Rayleigh wave measurement.

\section{MEASUREMENTS OF LSW VELOCITY BY FEM}

\subsection{Finite Element Modelling}

In this simulation model, piezoelectric polyvinylidene fluoride (PVDF) film is chosen as the active element and the tung- 


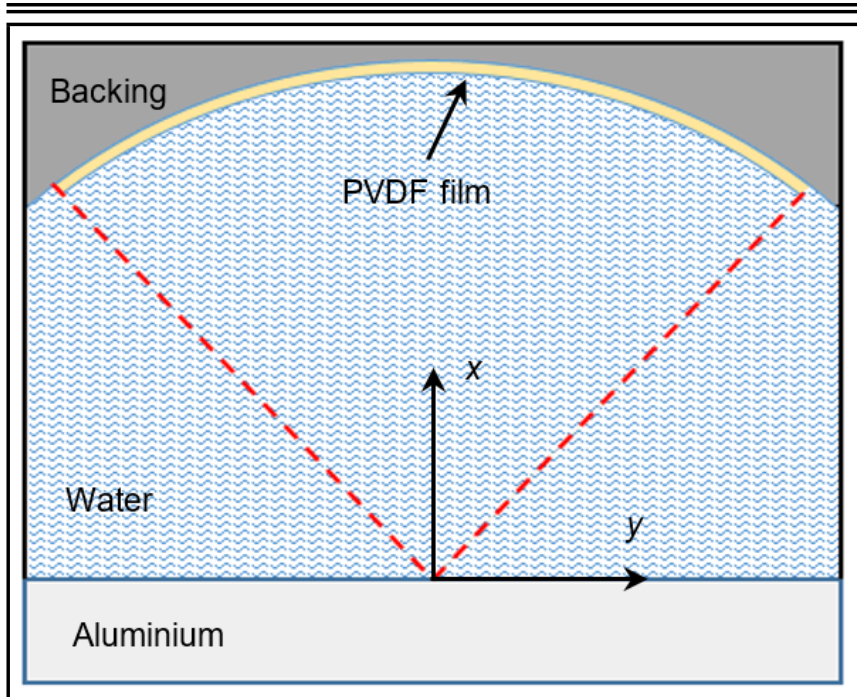

(a)

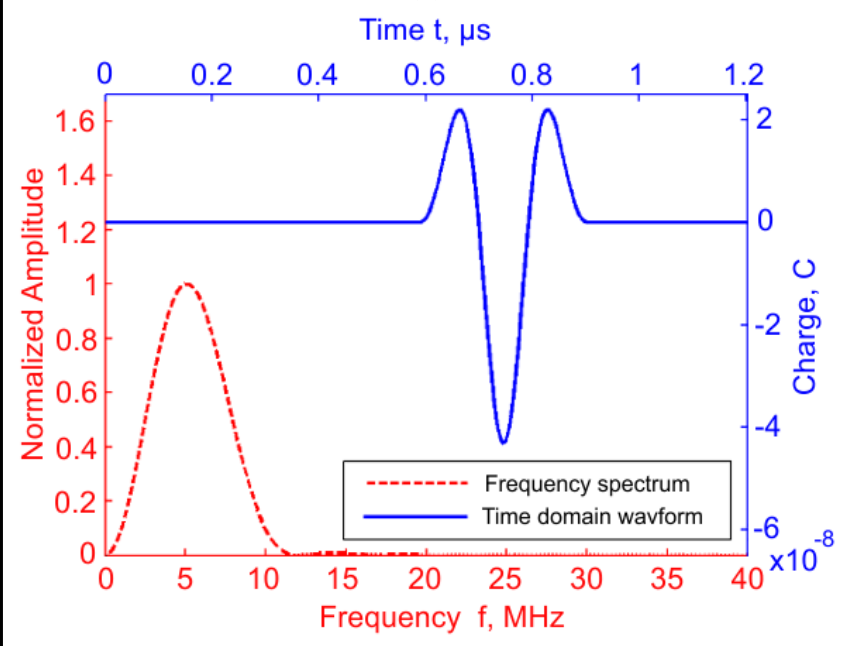

(b)

Figure 3. Finite element model and the time-frequency characteristic of excitation signalt.

sten loaded epoxy as backing. The line focus transducer is composed of backing and the PVDF film. The geometrical focusing radius, half aperture angle, and thickness of the PVDF membrane is $20 \mathrm{~mm}, 40^{\circ}$, and $40 \mu \mathrm{m}$, respectively. It should be noted that PZFlex uses brick-shaped Cartesian elements to construct the model. So, this finite element model employs the square-shaped elements to mesh the grid. The thickness of the aluminium specimen is $5 \mathrm{~mm}$. Since a symmetry plane is assumed at the $y=0$, only half of the physical geometry need to be included in the model. Figure $3 \mathrm{a}$ shows the 2-D finite element model for defocus measurement. Figure $3 \mathrm{~b}$ shows the excitation pulse, which is a black Harris wavelet (BHW), and its FFT spectrum.

Suitable wave propagation time and element size are important for the accuracy of numerical solutions in finite element calculations involving wave propagation. In this work, the actual wave propagation time is set to $35 \mu \mathrm{s}$. The model is discretized with 50 elements per wavelength for the longitudinal wave in water at $5 \mathrm{MHz}$. This results in a model composed of 4000 and 2250 elements in the $\mathrm{x}$ - and y-direction, respectively. The whole model contained a total of about 10 million elements. Furthermore, boundary conditions and material properties must realistically represent the experimental situation. The symmetry plane was at $y=0 \mathrm{~mm}$. The right $(y=13.5 \mathrm{~mm})$ and the top ( $x=21 \mathrm{~mm}$ ) boundary of the model was set to absorption boundary condition in order to prevent any reflections, and the bottom $(x=-5 \mathrm{~mm})$ of the model was set to free boundary condition.

The top (to backing) and the lower (to water) surface of the PVDF film is the positive and the negative electrode separately. In the simulation, the PVDF film is first excited. Then a transient pulse is launched into the coupling water and focused on the sample surface subsequently, which is also the focal plane of the transducer. When the transducer moves downward, the focal plane will separate from the sample surface, also known as defocusing. The wave beams incident at the Rayleigh angle are converted into surface wave. While this surface wave propagates along the sample surface, it leaks into the coupling fluid and re-radiates back to the PVDF transducer. In this case, the echo signals consist of not only the directly reflected waves and the bottom reflected longitudinal waves, but also the leaky surface waves. Figure 4 shows the wave propagation process when the waves are at different moments.

\subsection{Time-Resolved Method}

To sum up all the considerations mentioned above, the LSW velocities of aluminium are measured by this finite element model. First, the sample surface is aligned with the geometric focal plane, and the acoustic waves launched by the PVDF film are reflected by the sample surface. The received waves will be converted into charge signals by the top electrode of the PVDF film. Then the defocusing position $z$ steps continuously with $0.2 \mathrm{~mm}$ from $z=2 \mathrm{~mm}$ to $z=4 \mathrm{~mm}$.

Figure 5 shows the defocusing waveforms superposed by offsetting from different defocusing distances. The peaks in each waveform are extracted, and linear fitting of peak points brings out the slope of the arrival time of surface waves. Substituting the slope $m$ and $C_{w}=1496 \mathrm{~m} / \mathrm{s}$ into Eq. (1), one can get the LSW velocity of aluminium easily. Finally, the simulation result of LSW velocity is $3036 \mathrm{~m} / \mathrm{s}$ for aluminium.

\subsection{Analysis of Wave Propagation Path}

In Fig. 5, the DRE, LSW (re-radiated to the PVDF film), and the BRE can be clearly distinguished from the simulation signals in defocusing measurement. The LSWs' propagating path will be analyzed geometrically in time domain. The PVDF film is cut into 40 segments equally, i.e., one degree for an independent sensing segment. Figure 6 shows the half finite element model and the serial number of sensing elements (No.6 to No.34 are not illustrated). These multi-angle sensing elements are excited simultaneously, but they will receive the reflected wave separately. Compared with the PVDF film receiving the signal as a whole, in which the received signal is an integration of the reflected waves, these separated elements will obtain the detail information from each angle. In this model, $z=2 \mathrm{~mm}$ and $h=5 \mathrm{~mm}$ are given. The longitudinal wave velocity of aluminium is $6306 \mathrm{~m} / \mathrm{s}$. Figure 7 shows signals received by the multi-angle elements and the integrated signal. Meanwhile, the DREs' signals are firstly received by the centre element. The LSWs' will be received by the Rayleigh angle element at last among all the sensing elements. However, this is barely possible to observe in experiments.

The geometrically propagating path of DREs in time domain is shown in Fig. 8. The DREs' total propagating time from incident element by angle $\Theta_{1}$ (unknown variable) to receiving element by angle $\Theta$ is:

$$
T_{\mathrm{DRE}}=\frac{L_{\mathrm{AB}}+L_{\mathrm{BC}}}{C_{\mathrm{w}}} .
$$




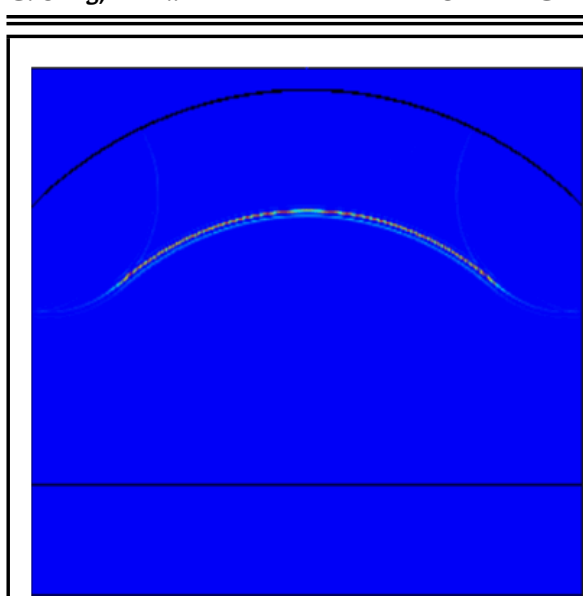

(a)

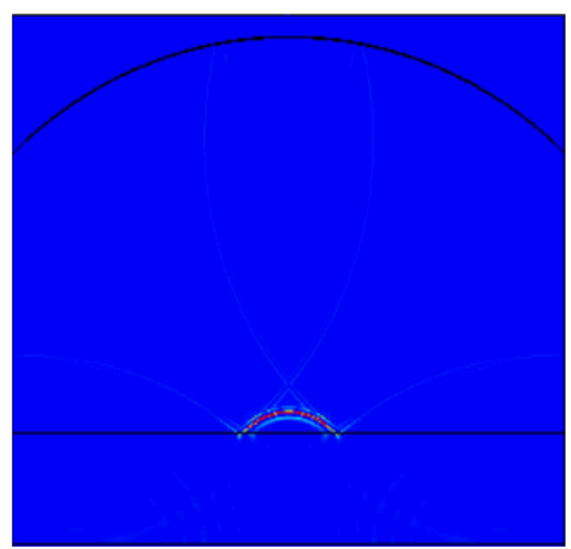

(b)

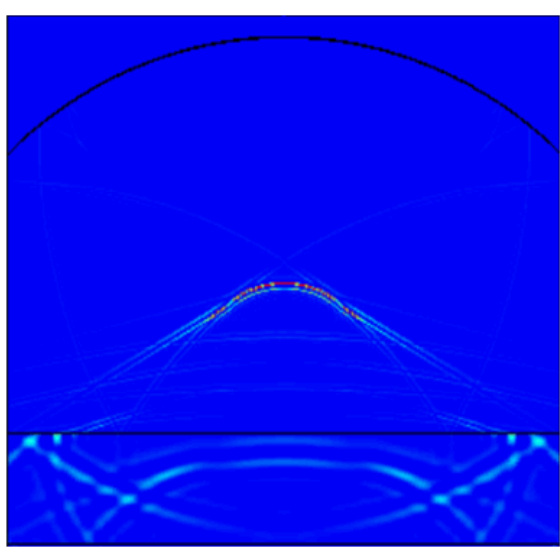

(c)

Figure 4. Snapshot of wave propagation when the transducer is placed at a defocused position: (a) $t=3 \mu \mathrm{s}$; (b) approaching the sample surface; (c) reflected waves.

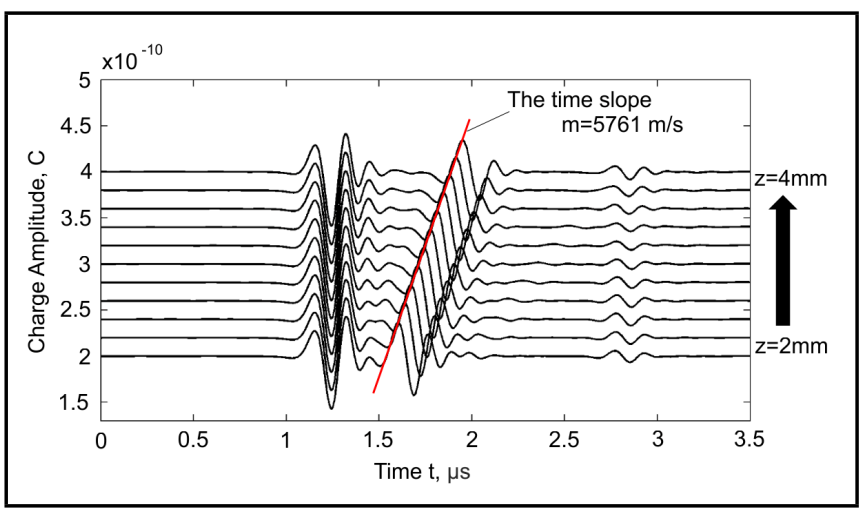

Figure 5. Time domain waveform.

The incident distance of DREs' propagating path LAB can be expressed as:

$$
L_{\mathrm{AB}}=R-\frac{z}{\cos \theta_{1}} .
$$

In triangle $\triangle B O C, L_{B O}=z\left(\cos \Theta_{1}\right)$ and $\Theta_{2}=2 \times\left(90^{\circ}-\right.$ $\left.\Theta_{1}\right)$. According to the law of sines, the distance of the reflected wave from the sample surface should be:

$$
L_{\mathrm{BC}}=\frac{R \sin \left(2 \times \theta_{1}-\arcsin \left(\frac{2 \times z \sin \theta_{1}}{R}\right)\right)}{\sin \left(2 \times \theta_{1}\right)} .
$$

Substituting Eq. (9) and Eq. (10) into Eq. (8), one can get the DREs' total propagating time easily. Meanwhile, the angle of the receiving element is:

$$
\theta=\theta_{1}-\arcsin \left(\frac{2 \times z \sin \theta_{1}}{R}\right) .
$$

The geometrical propagating path of LSWs are shown in Fig. 9. The LSWs' total propagating time from incident element by Rayleigh angle $\Theta_{R}$ to receiving element by angle $\Theta$ is:

$$
T_{\mathrm{LSW}}=\frac{L_{\mathrm{AB}}+L_{\mathrm{CD}}}{C_{\mathrm{w}}}+\frac{L_{\mathrm{BC}}}{C_{\mathrm{R}}} .
$$

The incident distance of LSWs' propagating path is the same as the DRE condition, which is:

$$
L_{\mathrm{AB}}=R-\frac{z}{\cos \theta_{\mathrm{R}}} .
$$

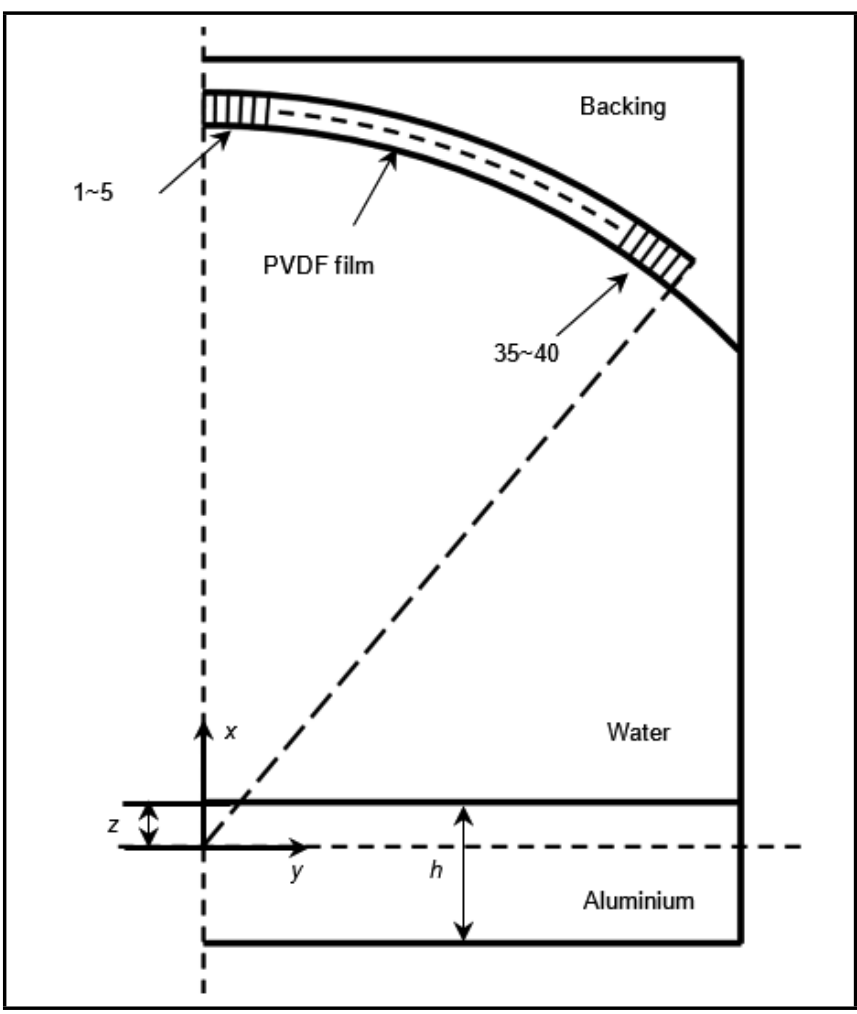

Figure 6. Half of the finite element model.

The distance $L_{B C}$ (unknown variable) of LSWs' propagating on the interface ought to be divided into three cases:

1. $L_{B C} \leq z \tan \left(\Theta_{R}\right)$

In triangle $\triangle D C E, L_{B O}=z \tan \left(\Theta_{R}\right)$, then $L_{C E}=$ $\sqrt{\left(\left(L_{B O}-L_{B C}\right)^{2}+z^{2}\right.}$ and $\Theta_{2}=90^{\circ}-\Theta_{R}+$ $\arctan \left[z\left(L_{B O}-L_{B C}\right)\right]$. According to the law of sines, the distance of reflected wave from the top of sample to multi-angle elements can be expressed as:

$$
L_{\mathrm{CD}}=\frac{R \sin \left(\theta_{2}+\arcsin \left(\frac{L_{\mathrm{CE}} \sin \theta_{2}}{R}\right)\right)}{\sin \theta_{2}} .
$$

Substituting Eq. (13) and Eq. (14) into Eq. (12), one can get the DREs' total propagating time easily. Meanwhile, the angle of the receiving element is: 


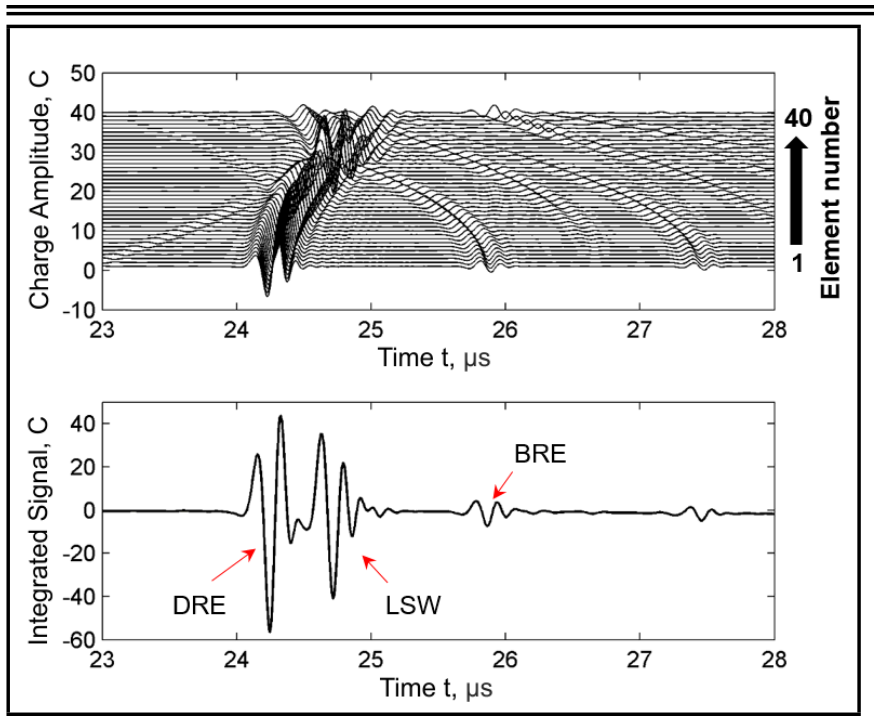

Figure 7. Signals received by multi-angle elements and its integration.

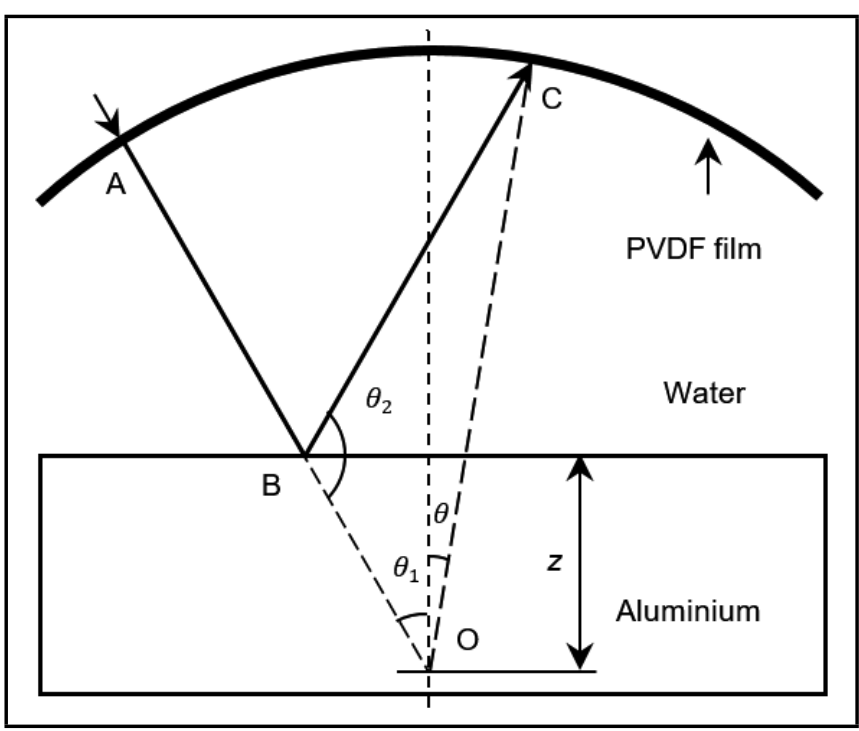

Figure 8. Geometrical illustration of the DREs' propagating path.

$$
\begin{aligned}
\theta=180^{\circ}-\theta_{2}-\arcsin \left(\frac{L_{\mathrm{CE}} \sin \theta_{2}}{R}\right)- \\
-\arctan \left(\frac{L_{\mathrm{BO}}-L_{\mathrm{BC}}}{z}\right) .
\end{aligned}
$$

2. $z \tan \left(\Theta_{R}\right) \leq L_{B C} \leq 2 \times z \tan \left(\Theta_{R}\right)$

In triangle $\triangle D C E, L_{B O}=z \tan \left(\Theta_{R}\right), L_{C E}=$ $\sqrt{\left(L_{B C}-L_{B O}\right)^{2}+z^{2}}$ and $\Theta_{2}=270^{\circ}-\Theta_{R}-$ $\arctan \left(z\left(L_{B C}-L_{B O}\right)\right)$. Also, according to the law of sines, the propagating distance of the reflected wave can be expressed as:

$$
L_{\mathrm{CD}}=\frac{R \sin \left(\theta_{2}+\arcsin \left(\frac{L_{\mathrm{CE}} \sin \theta_{2}}{R}\right)\right)}{\sin \theta_{2}} .
$$

Substituting Eq. (13) and Eq. (16) into Eq. (12), the DREs' total propagating time will be obtained. Then, the angle of the receiving element is:

$$
\begin{aligned}
\theta=180^{\circ}-\theta_{2}-\arcsin \left(\frac{L_{\mathrm{CE}} \sin \theta_{2}}{R}\right)+ \\
+\arctan \left(\frac{L_{\mathrm{BC}}-L_{\mathrm{BO}}}{z}\right) .
\end{aligned}
$$

3. $L_{B C} \geq 2 \times z \tan \left(\Theta_{R}\right)$

The same applies to the third case, that in triangle $\triangle D C E, \quad L_{B O}=z \tan \left(\Theta_{R}\right), L_{C E}=$ $\sqrt{\left(L_{B C}-L_{B O}\right)^{2}+z^{2}}$ and $\Theta_{2}=90^{\circ}+\Theta_{R}+$ $\arctan \left(z\left(L_{B C}-L_{B O}\right)\right)$. The reflected ray will propagate in:

$$
L_{\mathrm{CD}}=\frac{R \sin \left(\theta_{2}+\arcsin \left(\frac{L_{\mathrm{CE}} \sin \theta_{2}}{R}\right)\right)}{\sin \theta_{2}} .
$$

Substituting Eq. (13) and Eq. (18) into Eq. (12), the DREs' total propagating time is extracted, and the angle of the receiving element is:

$$
\begin{aligned}
\theta=\arctan ( & \left.\frac{L_{\mathrm{BC}}-L_{\mathrm{BO}}}{z}\right)+\theta_{2}+ \\
& +\arcsin \left(\frac{L_{\mathrm{CE}} \sin \theta_{2}}{R}\right)-180^{\circ} .
\end{aligned}
$$

Figure 10 shows the geometrical propagating path of BREs. The BREs' total propagating time from incident element by angle $\Theta_{1}$ to receiving element by angle $\Theta$ is:

$$
T_{\mathrm{BRE}}=\frac{L_{\mathrm{AB}}+L_{\mathrm{DE}}}{C_{\mathrm{w}}}+\frac{2 \times L_{\mathrm{BC}}}{C_{\mathrm{L}}} ;
$$

where $C_{L}$ is the longitudinal wave velocity of aluminium. Also, for BREs, the incident distance is:

$$
L_{\mathrm{AB}}=R-\frac{z}{\cos \theta_{1}} .
$$

According to the Snell's Law, the angle of refraction

$$
\theta_{2}=\arcsin \left(\frac{C_{\mathrm{L}} \sin \theta_{1}}{C_{\mathrm{w}}}\right) .
$$

So we have:

$$
\begin{gathered}
L_{\mathrm{BC}}=\frac{h}{\cos \left(\arcsin \left(\frac{C_{\mathrm{L}} \sin \theta_{1}}{C_{\mathrm{w}}}\right)\right)} \\
L_{\mathrm{BD}}=h \tan \left(\arcsin \left(\frac{C_{\mathrm{L}} \sin \theta_{1}}{C_{\mathrm{w}}}\right)\right) .
\end{gathered}
$$

In triangle $\triangle E F D, L_{D F}=\sqrt{\left(L_{B D}-z \tan \Theta_{1}\right)^{2}+z^{2}}$ and $\Theta_{3}=90^{\circ}+\Theta_{1}+\arcsin \left(z L_{D F}\right)$. According to the law of sines, the distance of reflected wave from the interface to multi-angle elements can be expressed by:

$$
L_{\mathrm{DE}}=\frac{R \sin \left(\theta_{3}+\arcsin \left(\frac{L_{\mathrm{DF}} \sin \theta_{3}}{R}\right)\right)}{\sin \theta_{3}} .
$$

Substituting Eq. (21), Eq. (23), and Eq. (25) into Eq. (20), one can get the BREs' total propagation time, and the angle of the receiving element is:

$\theta=\arccos \left(\frac{z}{L_{\mathrm{DF}}}\right)+\theta_{3}+\arcsin \left(\frac{L_{\mathrm{DF}} \sin \theta_{3}}{R}\right)-180^{\circ}$. 


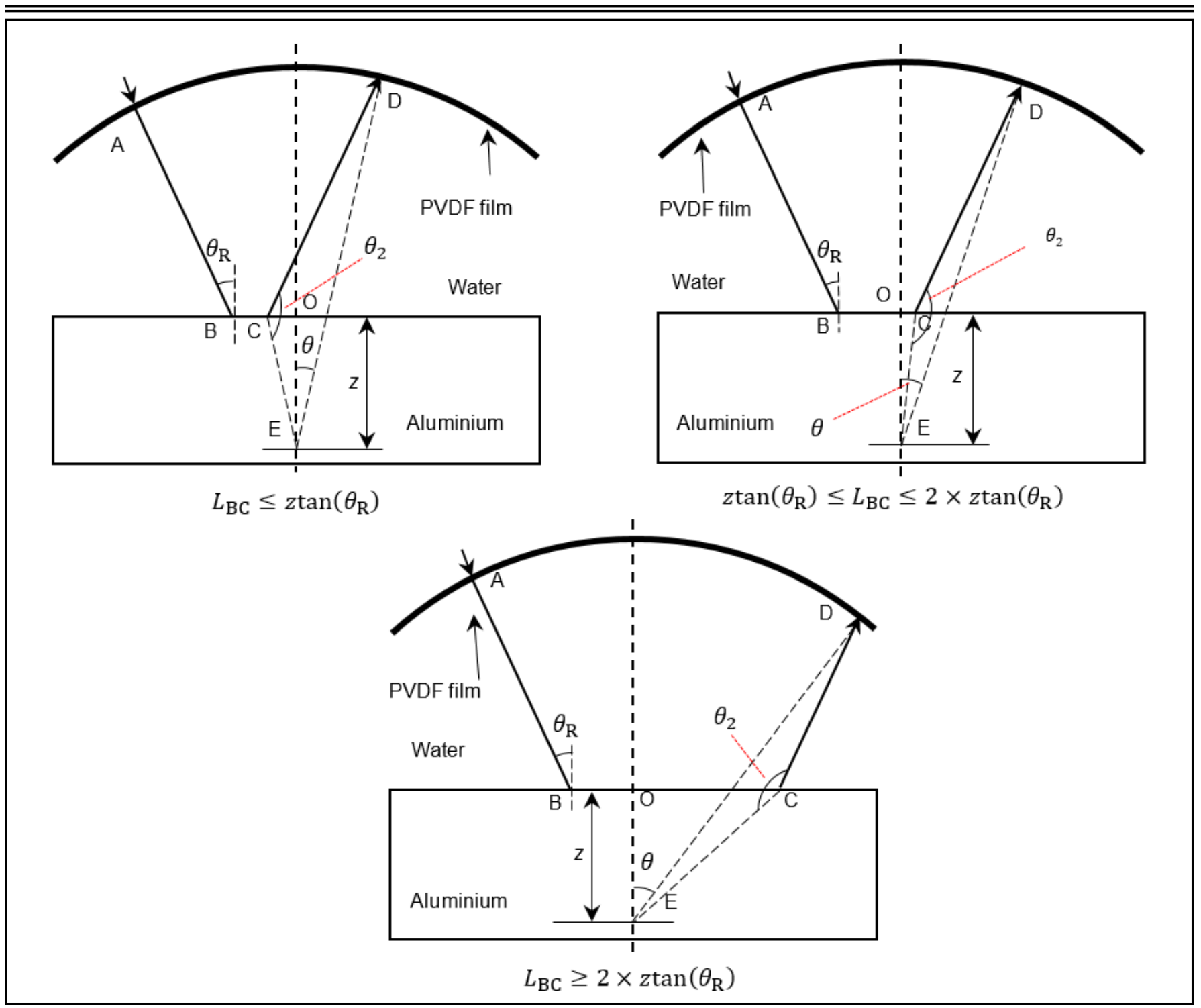

Figure 9. Geometrical schematic of the LSWs' propagating path.

In this section, to be clear:

(1) In DREs and BREs, if $\Theta_{1}$ is given, the propagating time $T_{D R E}$ and $T_{B R E}$ will be determined;

(2) In LSWs, the surface wave will leak to the water as it propagates, which means only $L_{B C}$ varies. Thus, the corresponding element (element $\Theta$ ) will receive a series of varying signals, as shown in Fig. 11.

The arriving time of DREs, LSWs, and BREs are shown in red solid line, blue dash line, and pink dash line, respectively, in Fig. 11, in which we could see their variation clearly. It should be noted that, the receiving angle $\Theta_{R}$ is always the last one to collect the LSW signal, and this feature can be used to determine the Rayleigh angle of the specimen. This geometrical analysis result is compared with the simulation. There is a good agreement between the results and the simulation. Finally, the integrated time domain waveform, like Fig. 7b, is able to be decomposed into multi-angle signals. In this process, the formation of the integrated signal appears clearly.

\section{4. $V(f, z)$ Analysis Method}

According to the $V(f, z)$ analysis method, ${ }^{18}$ the defocusing distance and interval here are $4 \mathrm{~mm}$ and $0.04 \mathrm{~mm}$, respec- tively. Thus, 101 groups of simulation waveforms are acquired. It should be mentioned that each of the waveforms is shifted in time domain so that the arrival time of the DRE is coincident to the original time reference. The waveform amplitudes will be normalized with respect to the magnitude of the DRE. Figure 12a shows all the normalized waveforms from top view, while Fig. 5 shows some equally selected ones. In order to obtain oscillation periods of the defocusing distance at different frequencies, the normalized time domain waveforms $v(t, z)$ are Fourier transformed with respect to time $t$ into frequency domain $f$, hereinafter referred to as $V(f, z)$ data shown in Fig. 12b. As a matter of fact, they both are induced by the interference between the DRE and LSW. In order to determine the oscillating period $\Delta z$ in $V(f, z)$ curve accurately, the $V(f, z)$ data are Fourier transformed to wave number $k$ domain (which is in fact the inverse of $z$ ) with respect to $z$, referred to as $V(f, k)$ data shown in Fig. 13a. From Fig. 3b, it can be found that the excitation pulse centred at the $5 \mathrm{MHz}$. However, the frequency domain is extended to $20 \mathrm{MHz}$. Even if there is a little energy in the high frequency domain, the $V(f, z)$ method, which is a "normalized" method with respect to frequency, can extract the periodical oscillation in $\mathrm{z}$ direction, as shown in Fig. 12b. The oscillating period $\Delta z$ will be determined by tracing the maxima on the "ridge", and it can be 


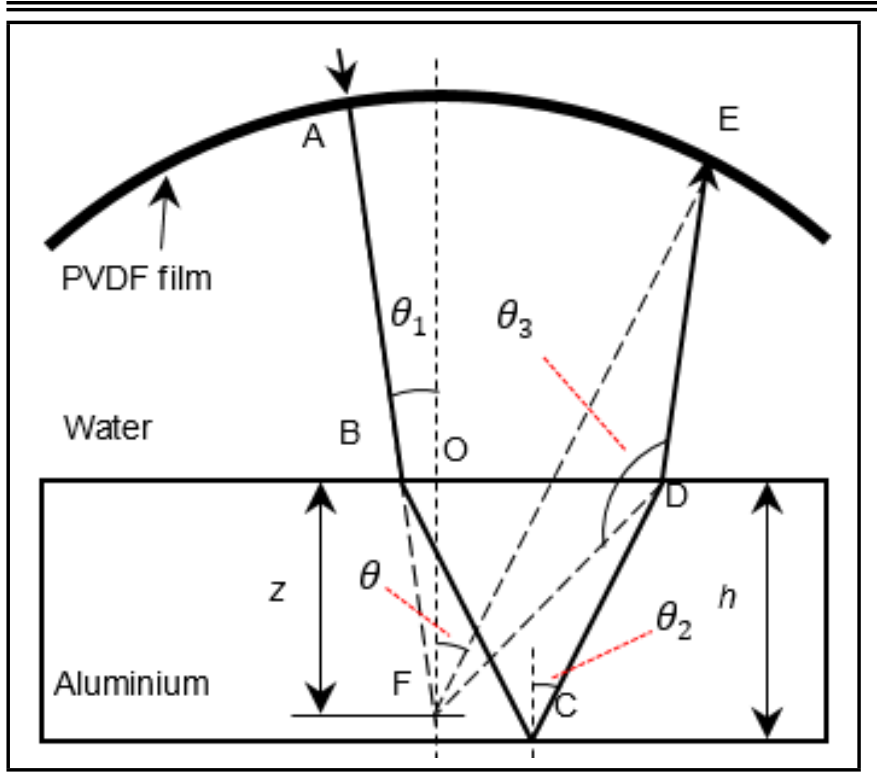

Figure 10. Geometrical schematic of the BREs' propagating path.

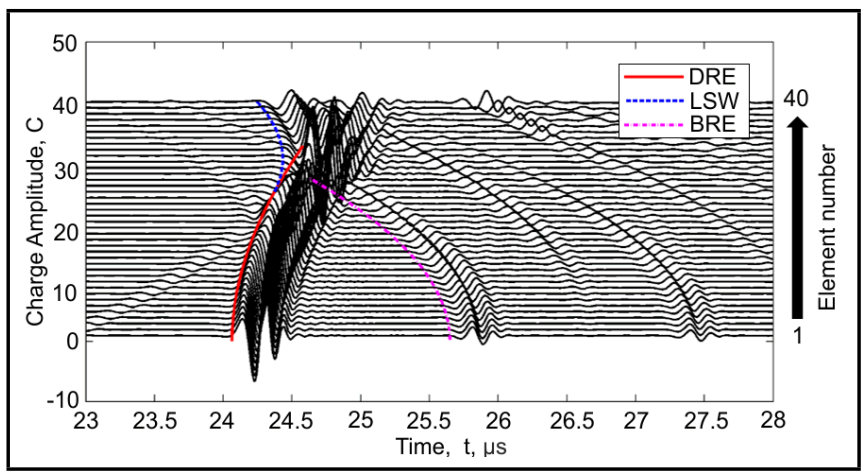

Figure 11. Signals received by multi-angle elements.

observed that the tracing range can extend from several $\mathrm{MHz}$ to over $15 \mathrm{MHz}$. Once the $\Delta z$ is determined, the SAW velocities will be obtained by Eq. (7), as shown in Fig. 13b. It appears that the velocities are very close to a constant from $4 \mathrm{MHz}$ to $10 \mathrm{MHz}$ with a discrepancy of less than $100 \mathrm{~m} / \mathrm{s}$. The simulation results of LSW velocity is $3045 \mathrm{~m} / \mathrm{s}$ by $V(f, z)$ technique.

\section{DISCUSSION}

This paper presents a FEM to simulate the lens-less line focus acoustic microscopy for non-destructive evaluation of the leaky surface wave (LSW) speed. The DRE, LSW reradiated to the PVDF film, and BRE are clearly time resolved in simulations of defocusing measurements on typical bulk materials (Al). More importantly, an analytical geometric model is established to illustrate the formation of the time-resolved waveform. From the multi-angle signal, the LSWs' propagating path is analyzed geometrically in time-domain. Also, this model reveals the mechanism of wave propagation in line focus acoustic field. Meanwhile, the time-resolved wave signal series are acquired when the defocusing distance varies continuously. Next, $V(f, z)$ analysis method is carried out and applied on these defocusing measurement data to obtain the surface wave velocity. The former simulation model shows the spatial analysis, so that one can understand how the LSW emerged from the DRE. The latter $V(f, z)$ analysis gives a time-frequency observation, so that the relationship of the time-resolved method and this $V(f, z)$ method can be linked

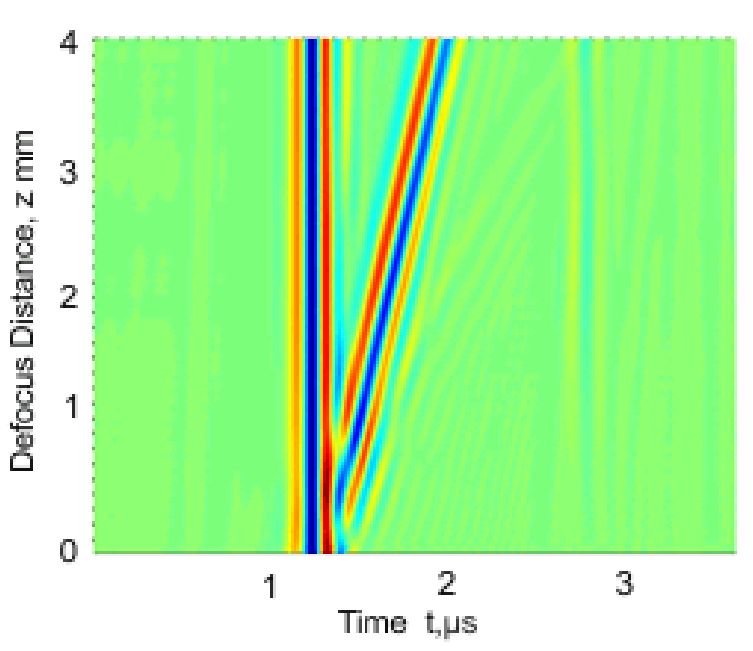

(a)

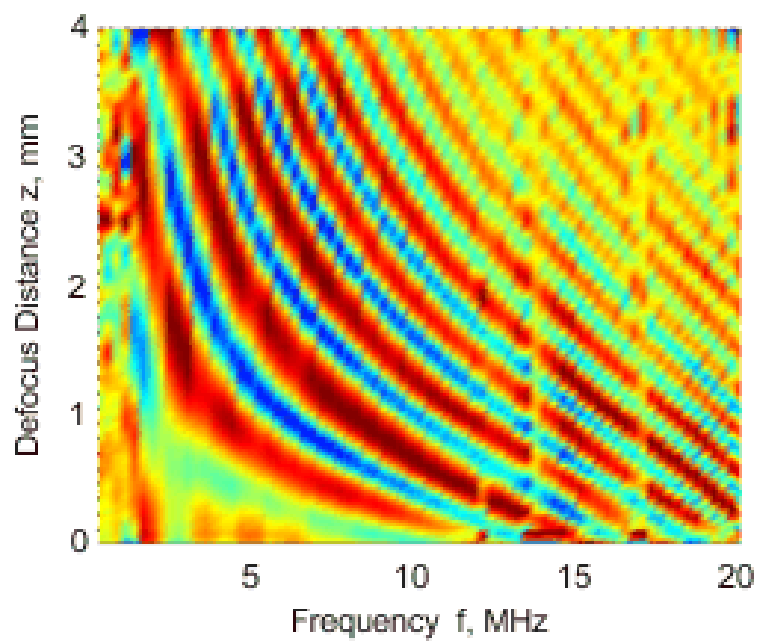

(b)

Figure 12. (a) Normalized waveforms of 3D-time; (b) Fourier transform with respect to $t$.

up. Furthermore, the multi-angle signal carries the structure's detailed information so that this method can be used to extract the characteristics (such as the orientation and depth) of the surface crack quantitatively.

\section{ACKNOWLEDGEMENTS}

The work in this paper is supported by the National Natural Science Foundation of China (Grant Nos. 61271372 and 51575015) and the Outstanding Young Researcher Program from Organization Department of the CPC Beijing Municipal Committee (Grant No. 2014000020124G038).

\section{REFERENCES}

1 Courant, R., Variational methods for the solution of problems of equilibrium and vibrations, Bulletin of the American Mathematical Society, 49, 123, (1943). https://dx.doi.org/10.1090/S0002-9904-1943-07818-4

2 Dongtao, Z., jiuxing, Z., and Meiling. Z., Advance in Bulk Nanocrystalline Materials, Metallic Functional Materials, 9(4), 1519, (2002). https://dx.doi.org/10.13228/j.boyuan 


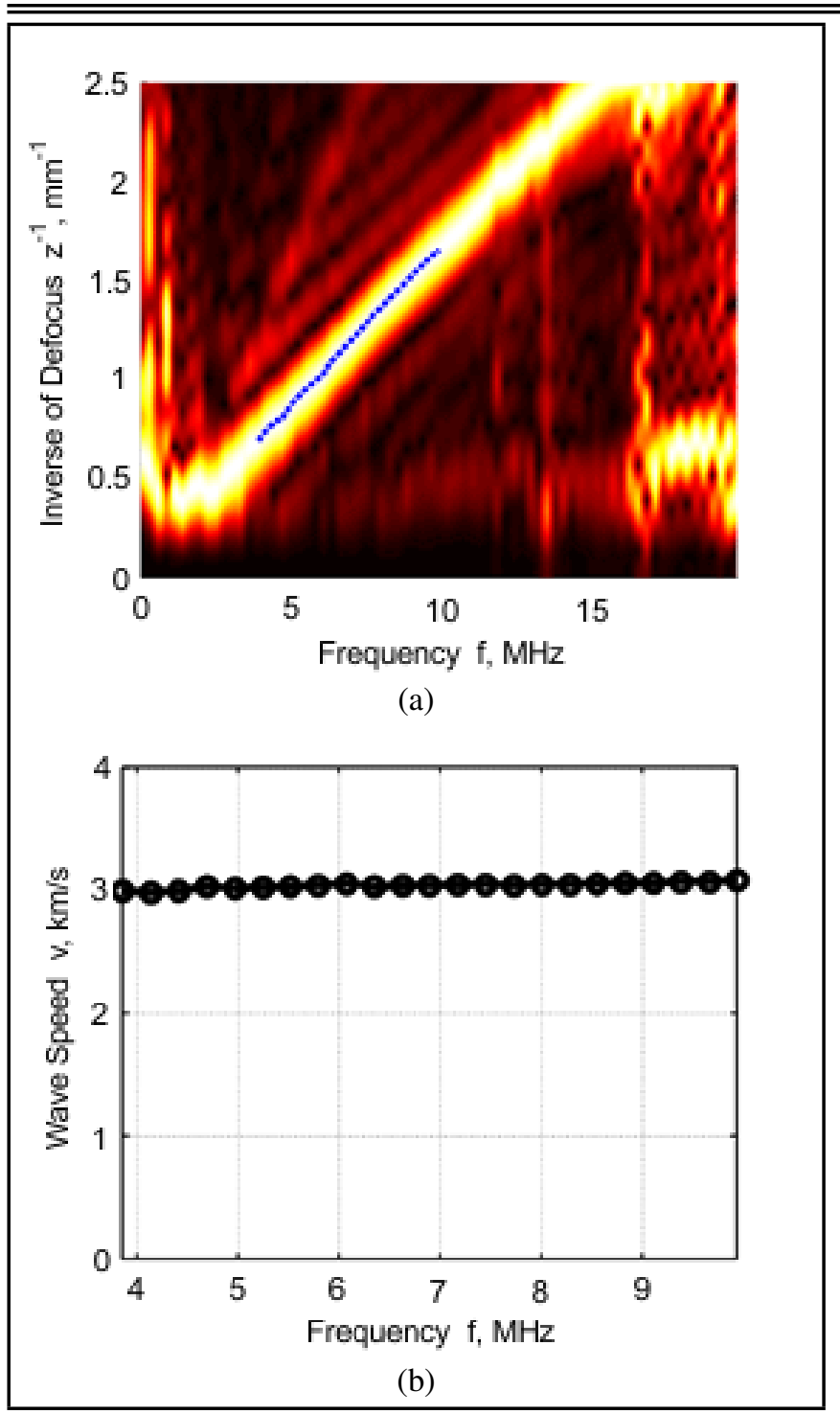

Figure 13. (a) Image representation of Fourier transform of $V(f, z)$ with respect to $z$; (b) measured surface wave speed.

3 Zhou Y. S., Wu, X. J., Xu, G. L., Li, B. H., Zhang, H.F., Du, L. G., and Li, Z. Q., Synthesis, microdefects and mechanical properties of large bulk nanocrystalline silver and copper. The Chinese Journal of Nonferous Metal, 10(4), 465469, (2000). https://dx.doi.org/10.3321/j.issn:1004-0609.2000.04.001

4 Comte, C., and Stebut, J. V. Microprobe-type measurement of Young's modulus and Poisson coefficient by means of depth sensing indentation and acoustic microscopy, Surface and Coatings Technology, 154(1), 4248, (2002). https://dx.doi.org/10.1016/S0257-8972(01)01706-6

5 Pastorelli, R., Ferrari, A. C., Beghi, M. G., Bottani, C. E., and Robertson, J., Elastic constants of ultrathin diamond-like carbon films, Diamond and Related Materials, 9, 825830,(2000). https://dx.doi.org/10.1016/S09259635(99)00245-9

${ }^{6}$ Zhang H. X., Zhang T. H., and Huan Y., Nanoindentation and nanoscratch measurements on the mechanical properties of $\mathrm{SiO}_{2}$ film, $\mathrm{Mi}$ cronanoelectronic Technology, 245248, (2003). https://dx.doi.org/10.13250/j.cnki.wndz.2003.z1.073
7 Bamber, M. J., Cooke, K. E., Mann, A. B., and Derby, B., Accurate determination of Young's modulus and Poisson's ratio of thin films by a combination of acoustic microscopy and nanoindentation, Thin Solid Films, 398, 299-305, (2000). https://dx.doi.org/10.1016/S00406090(01)01341-4

8 Atalar, A., Quate, C. F., and Wickramasinghe, H. K., Phase imaging in reflection with the acoustic microscope, Applied Physics Letters, 31, 791793, (1977). https://dx.doi.org/10.1063/1.89551

9 Weglein, R. D., and Wilson, R. G., Characteristic material signatures by acoustic microscope, Electronics Letters, 14 (12), 352354, (1978). https://dx.doi.org/10.1049/el:19780238

10 Parmon, W., Bertoni, H. L., Ray interpretation of the material signature in the acoustic microscope, Electronics Letters, 15(21), 684686, (1979). https://dx.doi.org/10.1049/el:19790486

11 Kushibiki, J., Chubachi, N., Material Characterization by Line-Focus-Beam Acoustic Microscope, IEEE Transactions on Sonics and Ultrasonics, 32 (2), 189212, (1985). https://dx.doi.org/10.1109/T-SU.1985.31586

12 Xiang, D. Hsu, N. N., and Blessing, G. V., The design, construction and application of a large aperture lens-less line-focus PVDF transducer, Ultrasonics, 34(6), 641647, (1996). https://dx.doi.org/10.1016/0041-624X(96)00058-3

13 Song, G. He., C., Liu, Z., Huang, Y., and Wu, B., et al. Measurement of elastic constants of limitedsize piezoelectric ceramic sample by ultrasonic method, Measurement, 42 (8), 12141219, (2009). https://dx.doi.org/10.1016/j.measurement.2009.04.008

14 He C., Wei, X., Song, g., Bi, X., and Wu, B., Research on the Measurement of Elastic Constants of the Smaller-specimen Materials, China Mechanical Engineering, 17(16), 17381741, (2006). https://dx.doi.org/10.3321/j.issn:1004-132X.2006.16.022

15 Wang, L. G., Determination of elastic constants of composites by time-resolved acoustic microscopy, Ultrasonics, 37(4), 283289, (1999). https://dx.doi.org/10.1016/S0041624X(98)00067-5

16 Hsu, N. N., Xiang, D., and Blessing, G. V., Timeresolved ultrasonic body wave measurements of material anisotropy using a lensless line-focus transducer, 1998 IEEE Ultrasonics Symposium, 2, 12611264, (1998). https://dx.doi.org/10.1109/ULTSYM.1998.765068

17 Lee, Y. C., and Chu, C. C., A double-layered line-focusing PVDF transducer and $V(z)$ measurement of surface acoustic wave, Japanese Journal Applied Physics, 44(3), 14621467, (2005). https://dx.doi.org/10.1143/JJAP.44.146

18 Lee, Y. C., Measurements of dispersion curves of leaky Lamb waves using a lensless line-focus transducer, Ultrasonics, 39(4), 297306, (2001). https://dx.doi.org/10.1016/S0041-624X(00)00069-X 